NASA/CR-1998-206918

ICASE Report No. 98-10

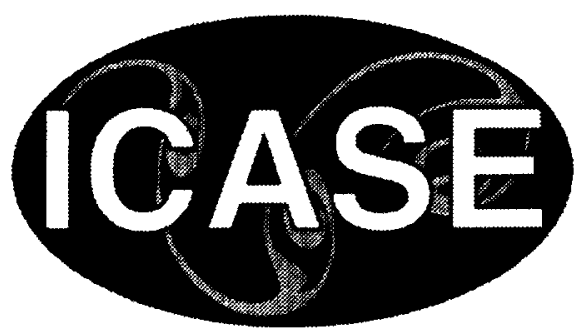

\title{
Numerical Study of Interaction of a Vortical Density Inhomogeneity with Shock and Expansion Waves
}

\author{
A. Povitsky \\ ICASE \\ D. Ofengeim \\ University of Manchester Institute of Science and Technology
}

Institute for Computer Applications in Science and Engineering NASA Langley Research Center

Hampton, VA

Operated by Universities Space Research Association

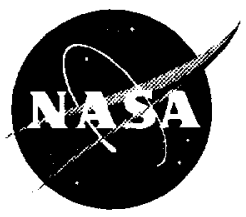

National Aeronautics and

Space Administration

Langley Research Center

Hampton, Virginia 23681-2199 
Available from the following:

NASA Center for AeroSpace Information (CASI) 800 Elkridge Landing Road

Linthicum Heights, MD 21090-2934

(301) 621-0390

National Technical Information Service (NTIS)

5285 Port Royal Road

Springfield, VA 22161-2171

(703) 487-4650 


\title{
NUMERICAL STUDY OF INTERACTION OF A VORTICAL DENSITY INHOMOGENEITY WITH SHOCK AND EXPANSION WAVES
}

\author{
A. POVITSKY * AND D. OFENGEIM ${ }^{\dagger}$
}

\begin{abstract}
We studied the interaction of a vortical density inhomogeneity (VDI) with shock and expansion waves. We call the VDI the region of concentrated vorticity (vortex) with a density different from that of ambiance. Non-parallel directions of the density gradient normal to the VDI surface and the pressure gradient across a shock wave results in an additional vorticity. The roll-up of the initial round VDI towards a non-symmetrical shape is studied numerically.

Numerical modeling of this interaction is performed by a 2-D Euler code. The use of an adaptive unstructured numerical grid makes it possible to obtain high accuracy and capture regions of induced vorticity with a moderate overall number of mesh points. For the validation of the code, the computational results are compared with available experimental results and good agreement is obtained.

The interaction of the VDI with a propagating shock wave is studied for a range of initial and induced circulations and obtained flow patterns are presented. The splitting of the VDI develops into the formation of a non-symmetrical vortex pair and not in a set of vortices.

A method for the analytical computation of an overall induced circulation $\Gamma_{1}$ as a result of the interaction of a moving VDI with a number of waves is proposed. Simplified, approximated, expressions for $\Gamma_{1}$ are derived and their accuracy is discussed.

The splitting of the VDI passing through the Prandtl-Meyer expansion wave is studied numerically. The obtained VDI patterns are compared to those for the interaction of the VDI with a propagating shock wave for the same values of initial and induced circulations. These patterns have similar shapes for corresponding time moments.
\end{abstract}

Key words. vortices, unstructured grids, automatic mesh refinement, Euler equations, vortical density inhomogeneity, shock wave, expansion wave

Subject classification. Fluid Mechanics

1. Introduction. A common situation in compressible flows is that regions of concentrated vorticity (vortex) are also the regions of inhomogeneous density, i.e, different density than ambiance. This vortical density inhomogeneity (VDI) interacts with either a shock or an expansion wave, i.e., with regions of pressure gradients. Current interest in this type of interaction is motivated to promote rapid mixing and combustion of hydrogen and hydrocarbon fuels for supersonic combustor applications and problems of direct numerical simulation of compressible turbulence. This research is a numerical investigation of this type of interaction.

Previous investigations of this type of interaction were concerned with the interaction of either a density inhomogeneity (initial vorticity is zero) [1] or a "pure" vortex [2] (without initial density inhomogeneity) with a shock wave.

Non-parallel directions of the density gradient normal to a VDI surface and the pressure gradient across

*This research was supported by the National Aeronautics and Space Administration under NASA Contract NAS1-97046 while the author was in residence at the Institute for Computer Applications in Science and Engineering (ICASE), NASA Langley Research Center, Hampton, VA, 23681-0001 (e-mail:aeralpo@icase.edu).

†Visiting researcher, Department of Mechanical Engineering, University of Manchester Institute of Science and Technology, on leave from Advanced Technology Center, P.O.Box 29, St. Peterburg 194156, Russia (e-mail:dima@telecom.lek.ru). 
a shock wave cause an additional vorticity (denoted also as induced vorticity). This vorticity causes the VDI to roll up into a non-symmetrical shape and to evolve towards a set of vortices of a finite core size. Detailed experiments were carried out by Jacobs [3] to quantify the mixing induced by the interaction of a shock wave with a cylindrical volume of a light gas (helium). Yang et al. [4] obtained an approximate analytical expression for this type of interaction.

A "pure" vortex interaction with a shock wave has been studied in various articles including the detailed numerical research reported in [5]. The experimental research [6] has shown that the vortex is somewhat compressed by the shock, while it generally retains the same configuration.

However, the interaction of the VDI with a shock wave as well as splitting of the VDI due to its passage through regions of pressure gradients, like expansion waves, has not been studied previously.

Generally, the presence of an induced and initial circulation can lead to the roll-up of the VDI into non-regular shapes, commonly including a number of vortices of different intensity. Our computations show that vortices of the same sign merge into a single vortex and VDI shapes transform into a non-symmetrical vortex pair of finite core size.

Our computations of the VDI interaction with an expansion wave show that in spite of obvious differences between interactions of VDI with shock and expansion waves, similar VDI shapes are obtained for the same values of induced and initial circulation. A correct estimation of induced circulation for the interaction with an expansion wave should be done. In this article, we derive it for the Prandtl-Meyer expansion wave.

Special numerical techniques were used by various authors for capturing the roll-up of a gas bubble moving with the flowfield after the shock-bubble interaction. Yang et al [4] use a regular numerical grid and rectangular computational domain moving in time to track the developing vortex pair. Quirk and Karni [7] use the Adaptive Mesh Refinement (AMR) algorithm. The AMR algorithm employs hierarchical system of rectangular grids. A local solution at a previous time step is used to determine automatically where refinement is needed to resolve small-scale phenomena.

An unstructured grid Euler solver [8]-[11] with a local refinement based on a density gradient is used in this research. Its advantage is an automatic capture of thin regions of induced vorticity at the VDI boundary.

In section 2, governing equations and the computational method used are described. In section 3 , we investigate the phenomena of the VDI splitting due to the interaction with a propagating shock wave and obtain dynamics of VDI patterns for a range of induced and initial circulation. In the last section, we derive analytical estimations for induced circulation due to the VDI interaction with the Prandtl-Meyer expansion wave and obtain VDI shapes by numerical computations.

2. Governing equations and numerical method. For a short limited time (a few milliseconds) viscous effects are minor and a non-viscous assumption is used to describe the phenomenon [4], [7].

The two-dimensional unsteady Euler equations of gas dynamics describing an inviscid compressible flow in conservative form can be written as:

$$
\boldsymbol{u}_{t}+\boldsymbol{f}_{x}+\boldsymbol{g}_{y}=0
$$

The vectors in equation (1) are

$$
\boldsymbol{u}=\left(\begin{array}{c}
\rho \\
\rho u \\
\rho v \\
e
\end{array}\right), \boldsymbol{f}=\left(\begin{array}{c}
\rho u \\
\rho u^{2}+p \\
\rho u v \\
(e+p) u
\end{array}\right), g=\left(\begin{array}{c}
\rho v \\
\rho u v \\
\rho u^{2}+p \\
(e+p) v
\end{array}\right)
$$


where $\rho$ is density, $p$-static pressure, $(u, v)$ - velocity in Cartesian coordinates $(x, y)$, and $e$ is the total energy, related to the other variables by an equation of state which for a perfect gas is

$$
e=\frac{p}{(\gamma-1)}+\frac{1}{2} \rho\left(u^{2}+v^{2}\right)
$$

where $\gamma=1.4$ ratio of specific heats.

The inviscid calculations have been carried out using an Euler code [10]. The code combines advantages of the total variation diminishing (TVD) finite-volume schemes, unstructured grids and an unsteady local adaptation of the grid to the features of the flow. The second-order Godunov-type scheme modified for transient flows and unstructured grids by Fursenko et al. [9], was employed to solve the Euler equations. This scheme is based on the TVD approach combined with second-order upwind differences and a solution of the Riemann problem to obtain inter-zone fluxes. More detailed description of the numerical method used in the Euler code can be found in $[8]-[9]$.

The unstructured grids are composed of triangular area elements, that provide a more isotropic spatial discretization as compared to traditional rectangular grids [8]. In order to generate an unstructured grid in the computational domain the 2-D unstructured grid generator created by Voinovich and Galyukov [11] was used. Fragments of the unstructured grid for a typical VDI interaction with a propagating shock wave are shown in Figure 1a-b. The distance between the neighboring grid points is $2^{k}$ times smaller than the initial distance. Computations are done with the maximum $\mathrm{k}$ equal to 5 .

The graphical presentation of numerical results has been done by the grid data VIGIE code [12] that allows to visualize an unstructured grid computed fluid flow.

There are no available experiments for interactions with the VDI; therefore, results of an experimental research for the interaction of a moving shock wave with density inhomogeneity [3] are used for the code verification. Experiments were carried out for the interaction of a shock wave $(M=1.09)$ with a cylindrical volume of gas (helium) that is lighter than ambiance (air). Computational results are presented in Fig 2. There is a good agreement with experimental results [3] at corresponding time moments.

3. Interaction between vortical density inhomogeneity and a propagating shock wave. A computational domain for the interaction between the VDI and a straight propagating shock wave is shown in Figure 3.

The initial conditions behind a shock wave are calculated according to the Rankine-Hugoniot relations; ahead of the shock wave ambient conditions are given. The shock wave proceeds into ambient conditions and interacts with a vortex superimposed on the ambient state.

The initial density ratio $\left(D=\rho_{l} / \rho_{h}\right)$ between a light VDI material $\left(\rho_{l}\right)$ and a heavy ambient air $\left(\rho_{h}\right)$ and the velocity ratio $\left(W=U_{c} / U_{b}\right)$ between a maximum tangential velocity $\left(U_{c}\right)$ and a velocity behind the shock wave $\left(U_{b}\right)$ are governing parameters that determine the dynamics of the VDI shape after an interaction with a propagating shock wave.

The 2-D vortex model [5] used for the VDI consists of two regions of the vortical flow: an inner core region $\left(R_{c}\right)$ and a surrounding region $\left(R_{o}\right)$ where the velocity gradually reduces to zero (see Fig. $3 \mathrm{a}$ ). In the numerical experiments carried out the linear tangential velocity profile in the VDI core region is considered and is represented by

$$
U_{\theta}(r)=U_{c} \frac{r}{R_{c}} ; r<R_{c}
$$


the outer distribution is given by

$$
U_{\theta}(r)=A r+\frac{B}{r}, R_{c} \leq r \leq R_{o}
$$

where $U_{\theta}$ is the tangential velocity, $U_{c}-$ maximum core velocity, $r=$ distance from vortex center, $R_{c}-$ vortex core radius ( $R_{c}=0.5 R_{o}$ is used), $R_{o}$ - outer radius.

The constants $\mathrm{A}$ and $\mathrm{B}$ are chosen in such a way that the velocity matches at $r=R_{c}$ and decays to zero at $r=R_{0}$. The initial circulation $\Gamma_{0}$ is equal to $2 \pi R_{c} U_{c}$. The vortex rotates counterclockwise.

An initial uniform density profile is taken inside the VDI ( $\rho=\rho_{l}$ for $r \leq R_{o}$ ).

Results of computations for the case $D=0.2 ; W=1.0$ and Mach number of the shock wave $M=1.5$ are presented in Fig 4. Density isolines are shown at the time moments: $50 \mu \mathrm{s}, 70 \mu \mathrm{s}, 90 \mu \mathrm{s}, 110 \mu \mathrm{s}, 157 \mu \mathrm{s}$, $203 \mu s, 421 \mu s$. In order to visualize strengths of vortices, streamlines are drawn in Fig. 4e-h.

The induction of vorticity due to the interaction with a shock wave leads to stretching of the initial vortex in the $y$ direction. The interaction of the initial and induced vorticity results in further distortion of the initial round vortex and its transformation to a "snake-like" structure with a long "tail" and a round "head" (Fig 4h).

Streamlines in the upper part of Figs. 4e-f are far from a typical pattern of flux streamlines near a singlepoint vortex. Later, initial and induced co-rotating counter-clockwise vorticity regions merge into one region of concentrated vorticity (see Figs. $4 \mathrm{~g}-\mathrm{h}$ ). The second counter-clockwise vortex is weak and hardly seen on density isolines and streamlines. The streamlines become similar to those around a non-symmetrical vortex pair.

In order to examine the influence of governing parameters $W$ and $D$ on VDI patterns three cases are considered. The ratio $\Gamma_{1} / \Gamma_{0}$ is calculated for these cases and presented in Table 1.

TABLE 1

The VDI interaction with a propagating shock wave, $\Gamma_{0}$ initial circulation, $\Gamma_{1}$ induced circulation, $\Gamma_{0} / \Gamma_{0 A}$, and $\Gamma_{1} / \Gamma_{1 A}$ are ratios of initial and induced circulation to those in the case $A$

\begin{tabular}{|c|c|c|c|c|c|}
\hline case & $D=\rho_{l} / \rho_{h}$ & $W=U_{c} / U_{b}$ & $\Gamma_{0} / \Gamma_{0 A}$ & $\Gamma_{1} / \Gamma_{1 A}$ & $\Gamma_{0} / \Gamma_{1}$ \\
\hline A & 0.2 & 1.0 & 1 & 1 & 0.888 \\
B & 0.2 & 0.5 & 0.5 & 1 & 0.444 \\
$\mathrm{C}$ & 0.5 & 1.0 & 1 & 0.4 & 1.776 \\
\hline
\end{tabular}

In order to estimate the induced circulation due to interaction between a VDI and a shock wave, an approximate expression obtained for shock-bubble interaction [4] is used:

$$
\Gamma_{1} \approx \frac{4 R_{0}}{V_{s}} \frac{\Delta p}{\rho_{2}} \frac{\rho_{h}-\rho_{l}}{\rho_{l}+\rho_{h}}
$$

where $\rho_{2}$ and $V_{s}$ are values of density and stream velocity behind the shock wave, $\Delta p$ - pressure drop across the shock wave.

The above expression was derived under the assumption of a uniform density profile inside the bubble, i.e., the density gradient is only caused by the density difference between the bubble and ambiance.

However, the density profile inside the VDI is not uniform due to rotation, and we have to prove that the density gradient at the outward boundary of the VDI (at the inner side) is equal to zero. 
After a short time (prior to the interaction) the profiles of pressure and density inside the VDI become close to those given by equilibrium equations for an isentropic steady vortex:

$$
\frac{d p}{d r}=\frac{\rho U_{\theta}^{2}}{r}
$$

$$
\frac{p}{\rho^{\gamma}}=\frac{p_{a m b}}{\rho_{a m b}^{\gamma}}
$$

with the boundary condition $p_{1}\left(r=R_{o}\right)=p_{a m b}$, where the index 'amb' corresponds to the ambiance conditions either in front or behind the shock wave.

The radial density gradient is obtained from (8):

$$
\frac{d \rho}{d r} \sim p^{1 / \gamma-1} \frac{d p}{d r},
$$

At the outward boundary of the VDI $U_{\theta}=0$; therefore, the pressure gradient is equal to zero (see (7)). Thus, the density gradient is also equal to zero at the inner side of the VDI boundary.

Dimensionless coordinates $\left(x / R_{o} ; y / R_{o}\right)$ of centers of vortices, relative density $\left(\rho_{c} / \rho_{a m b}\right)$ and pressure $\left(p_{c} / p_{a m b}\right)$ at these centers in $T=421 \mu s$ are presented in Table 2. Local pressure minimums are taken as centers of vortices.

TABLE 2

VDI shapes, $T=421 \mu \mathrm{s}, M V$-main counter-clockwise vortex, SV1-clockwise vortex, SV2-second counter-clockurse vortex.

Case A

\begin{tabular}{|l|l|l|l|l|}
\hline & $x / R_{o}$ & $y / R_{o}$ & $\rho_{c} / \rho_{a m b}$ & $p_{c} / p_{a m b}$ \\
\hline MV & 7.053 & 0.26 & 0.283 & 0.866 \\
SV1 & 7.413 & 0.76 & 0.846 & 0.893 \\
SV2 & 1.818 & 0.518 & 0.926 & 0.930 \\
\hline
\end{tabular}

Case B

\begin{tabular}{|l|l|l|l|l|}
\hline & $x / R_{o}$ & $y / R_{o}$ & $\rho_{c} / \rho_{a m b}$ & $p_{c} / p_{a m b}$ \\
\hline MV & 6.82 & 0.37 & 0.244 & 0.884 \\
SV1 & 7.23 & 3.03 & 0.672 & 0.920 \\
SV2 & 5.45 & 0.13 & 0.917 & 0.993 \\
\hline
\end{tabular}

Case C

\begin{tabular}{|l|l|l|l|l|}
\hline & $x / R_{o}$ & $y / R_{o}$ & $\rho_{c} / \rho_{a m b}$ & $p_{c} / p_{a m b}$ \\
\hline MV & 6.71 & 0.386 & 0.415 & 0.788 \\
SV1 & 7.41 & 0.933 & 0.968 & 0.961 \\
SV2 & 5.48 & 0.213 & 0.971 & 0.956 \\
\hline
\end{tabular}

The overall strength of vortices characterized by $\Gamma_{0}+\Gamma_{1}$ is maximum in case A and minimum in case $\mathrm{C}$ (see Table 1). Thus, the rotational velocity induced by vortices also reduces from case $\mathrm{A}$ to $\mathrm{C}$. This rotational 
velocity is added to the flux velocity $U_{b}$ and influences the speed of the VDI. The $\mathrm{x}$-coordinate of the main vortex is maximum in case $A$ and minimum in case $C$.

Due to the process of the merge of two counter-clockwise vortices the y-coordinate of the main vortex is shifted up. This shift is most marked in case A.

Density values show that the core of the main vortex remains almost unmixed. The mixing is more complete for the clockwise vortex (SV1). The second counter-clockwise vortex (SV2) is mixed with ambiance and its density is close to the ambiance density.

The values of a local pressure minimum at the centers of vortices depend upon the rotational velocity and density inside the VDI (see (7)). The deeper pressure minimum in case $\mathrm{C}$ is explained by a 2.5-time higher initial density than that in cases A and B. In spite of a higher density level in case $\mathrm{C}$, the pressure minimum in the center of SV1 vortex for case $\mathrm{C}$ is less than in cases A and B. This is due to a smaller induced vorticity (see (6)) and a corresponding lower level of rotational velocity.

Results for $W=0.1,0.25,0.5,1.0$ and the same initial density of the VDI $D=0.2$, i.e., the computations are performed for a constant $\Gamma_{1}$ and various $\Gamma_{0}$ values, are presented. Density isolines for these cases are given in Fig 5a-d. The reduction of the initial rotational velocity leads to the formation of a more symmetrical shape of a rolled-up VDI. In the case of a small initial vortical velocity a nearly symmetrical vortex pair is obtained (Fig. 5a). For higher values of $W$ the VDI structure becomes non-symmetrical. Pressure isolines are shown in Fig 5e-h and a pair of two round vortices appears for all values of $W$.

In all cases considered in this section the splitting of the VDI developes into a non-symmetrical vortex pair.

\section{Interaction between VDI and expansion waves.}

4.1. Estimation of induced circulation. The interaction between a Prandtl-Meyer expansion wave and light vortical inhomogeneity is considered (Fig. 3b).

In a Prandtl-Meyer expansion wave streamlines diverge around a sharp convex corner. The mechanism of inducing vorticity due to a non-parallel gradient of density at the outer boundary of the vortex and gradient of pressure is relevant for the case of VDI interaction with an expansion wave. However, in this case the VDI passes through the region of pressure gradient and not through a steep pressure drop as in the previous case of the interaction with a propagating shock wave. Therefore, the induced vorticity grows gradually and this is an essential difference between the two types of interaction.

A search is made for an appropriate estimation of the circulation induced in a VDI passing through an expansion wave. An expansion wave or a part of it can be characterized by upstream and downstream Mach numbers $\left(M_{u}\right.$ and $M_{d}$, respectively). The approximation (6) was derived under the assumptions of stepwise pressure drop across the shock wave and a constant velocity of a shock propagation. For the interaction between the VDI and an expansion wave an approximate expression for an induced circulation is written as a sum of the circulation induced due to interactions with $n$ weak waves:

$$
\Gamma_{1}=\sum_{i=2}^{n}\left(\frac{4 R}{V \rho}\right)_{i}\left(p_{i}-p_{i-1}\right)\left(\frac{\rho_{h}-\rho_{l}}{\rho_{l}+\rho_{h}}\right)
$$

The variation of Mach number for all $\mathrm{n}$ waves is the same: ${ }^{1}$

$$
\Delta M=M_{i+1}-M_{i}=\frac{M_{d}-M_{u}}{n-1}
$$

\footnotetext{
${ }^{1}$ Variation of local angle is not the same for these $n$ waves
} 
$p_{i-1}$ and $p_{i}$ denote upstream and downstream pressure of the $i-t h$ wave, $R_{i}$ radius of the VDI in the interaction with the $i-t h$ wave, $V_{i}$ is the gas velocity upstream of the $i$ - th wave and $\rho_{i}$ is the gas density downstream of the $i-t h$ wave.

The last term $\left(\rho_{h}-\rho_{l}\right) /\left(\rho_{l}+\rho_{h}\right)$ is constant due to the fact that the density ratio $\rho_{h} / \rho_{l}$ remains the same for an isentropic expansion of gases with the same ratio of specific heats. The first term, $Q_{i}=R /(V \rho)_{i}$ is variable as the radius of the VDI, velocity and density of the mainstream vary through an expansion wave.

The normalized value of induced circulation can be written as:

$$
\overline{\Gamma_{1}} \approx \sum_{i=2}^{n} \frac{Q_{i}}{Q_{d}} \frac{\left(p_{i}-p_{i-1}\right)}{P_{d}},
$$

where $Q_{d}=(R /(V \rho))_{d}$ and $P_{d}$ are values of the parameter $\mathrm{Q}$ and pressure downstream from the expansion wave.

The first term $Q_{i} / Q_{d}$ in the above equation can be expressed as a function of Mach numbers $M_{i}$ and $M_{d}$ as follows. The radius of the VDI increases due to the expansion of streamlines. The distance between streamlines is inversely proportional to the mass flux, $V \rho$. Therefore, the above term is given by the following expression:

$$
\frac{Q_{i}}{Q_{d}}=\left(\frac{V_{d} \rho_{d}}{V_{i} \rho_{i}}\right)\left(\frac{R_{i}}{R_{d}}\right)=\left(\frac{V_{d} \rho_{d}}{V_{i} \rho_{i}}\right)^{2}
$$

Stream velocities are connected directly to Mach numbers:

$$
\frac{V_{d}}{V_{i}}=\frac{M_{d}}{M_{i}} \frac{c_{d}}{c_{i}}
$$

where c - speed of sound.

Substituting isentropic relations [13]

$$
\begin{gathered}
\frac{p_{d}}{p_{i}}=\left(\frac{1+[(\gamma-1) / 2] M_{i}^{2}}{1+[(\gamma-1) / 2] M_{d}^{2}}\right)^{\frac{\gamma}{\gamma-1}} \\
\frac{\rho_{d}}{\rho_{i}}=\left(\frac{1+[(\gamma-1) / 2] M_{i}^{2}}{1+[(\gamma-1) / 2] M_{d}^{2}}\right)^{\frac{1}{\gamma-1}} \\
\frac{c_{d}}{c_{i}}=\sqrt{\frac{T_{d}}{T_{i}}}=\left(\frac{1+[(\gamma-1) / 2] M_{i}^{2}}{1+[(\gamma-1) / 2] M_{d}^{2}}\right)^{\frac{1}{2}}
\end{gathered}
$$

in (13), we have

$$
\frac{Q_{i}}{Q_{d}}=\left(\frac{M_{d}}{M_{i}}\right)^{2}\left(\frac{1+[(\gamma-1) / 2] M_{i}^{2}}{1+[(\gamma-1) / 2] M_{d}^{2}}\right)^{\frac{\gamma+1}{\gamma-1}}
$$

The normalized induced circulation is computed by (12) vs. parameter $Z=M_{i} / M_{d}$ for $M_{d}=1.25,1.5$, 1.75, 2.0 and is shown in Fig 6a. Computations were performed in an "upstream" manner up to $M_{u}=1$, i.e, for $Z$ ranges from $1 / M_{d}$ to 1 . The number of $\mathbf{n}$ waves is taken equal to 500 . 
The computed induced circulation can be used for practical purposes. However, these numerical computations are somewhat cumbersome, especially if an inversed problem is to be solved, i.e., to find $M_{u}$ or $M_{d}$ for a required level of induced circulation. On the other hand, the expression (6) is also an approximation of circulation; thus, high accuracy of integration (10) is excessive.

If $Q_{i}$ is replaced by its approximation $Q_{a p p}$ independent of the $i-$ th wave, then $\Gamma_{1}$ is equal to $Q_{a p p}$ multiplied by the difference of upstream and downstream pressures of the expansion wave. For this purpose the function $Q_{i}(Z)$ is studied in detail. Graphs of $Q_{i} / Q_{d}$ vs the parameter $\mathrm{Z}$ are shown in Fig. $6 \mathrm{~b}$. One can see that it is a slightly convex function of $Z$ and the first term in the sum (12) can be approximated by the average value of $Q_{u}$ and $Q_{d}$ :

$$
\bar{\Gamma} \approx 0.5\left(Q_{u}+Q_{d}\right)\left(p_{u}-p_{d}\right)
$$

The use of this approximation leads to an overestimation of induced circulation. A relative error between an approximate normalized induced circulation (19) and its value computed by (12) is shown in Fig. 7. The error increases with the $M_{d} / M_{u}$ ratio and with $M_{d}$. For $Z>0.75$, a relative error is no more than $10 \%$.

A straightforward approximation of $\Gamma$ in the same form as (6)

$$
\bar{\Gamma} \approx \frac{R_{u}}{V_{u} \rho_{d}}\left(p_{u}-p_{d}\right)
$$

leads to an underestimation of induced circulation. A relative error of the above approximation is more than that for the previous approximation (19)) (compare families of curves a and b in Fig. 7).

4.2. Numerical study . The VDI splitting after its interaction with an expansion wave is studied numerically. The expansion wave is considered provides the same amount of induced vorticity as for the previously studied shock wave-VDI interaction. The upstream Mach number is taken equal to the Mach number of the propagating shock in the previous case $\left(M_{u}=1.5\right)$. The value of induced circulation is computed by (6). We choose an angle $\theta$ of the convex corner such as to produce the desired Prandtl-Meyer expansion wave. For a given value of induced circulation the value of $Q_{d}$ is obtained from the expression (19). The downstream Mach number $M_{d}$ is calculated from equation (18). The angle of the convex corner is computed from

$$
\theta=\nu\left(M_{d}\right)-\nu\left(M_{u}\right)
$$

where $\nu(M)$ is the Prandtl-Meyer function [13]. For example, for $M_{u}=1.5$ and the downstream Mach number $M_{d}=2.01$ we obtain $\theta=13^{0}$.

The distribution of rotational velocity is given by equations (4), (5). The maximum rotational velocity of the vortex is taken equal to $0.3 \mathrm{M}$. Thus, rotational velocity and initial circulation are the same as in the previous case of the VDI interaction with a propagating shock wave.

A numerical simulation of the interaction between the VDI and the Prandtl-Meyer expansion wave is performed as follows. First, the Euler equations are solved numerically to obtain a converged steady state for uniformed flow followed by a Prandtl-Meyer expansion wave. After this the VDI was introduced into the flowfield.

The computational domain and the VDI before the interaction are shown in Fig. 3b. Typical stages of the VDI interaction with the expansion wave and downstream are presented in Fig. 8. Similar patterns of the VDI shapes are observed after the interaction with shock and expansion waves. We recall that both 
initial and induced circulations are equal in these two cases. However, the time scale is different in these cases. The effective time of VDI splitting after the interaction with the Prandtl-Meyer expansion wave can be computed by $T_{\text {eff }}=T-T_{\text {exit }}$, where $T_{\text {exit }}$ is the time when the VDI just passes an expansion wave and get all induced vorticity from the interaction. Time should also be scaled by the mainstream velocity( 2.01 $\mathrm{M} / 0.6 \mathrm{M}=3.35$ ). The VDI shape after the interaction with a shock wave at time $T=110 \mu \mathrm{s}$ (Fig. 4e) is compared with the VDI shape after interaction with the Prandtl-Meyer wave at the corresponding time moment $T_{\text {eff }}=110 * 3.35+371=740 \mu$ s (Fig. $8 \mathrm{~h}$ ). One can conclude that the vortical shapes are similar at corresponding times.

5. Conclusions. A numerical modeling of interaction between vortical density inhomogeneity (VDI) and shock and expansion waves was performed using a 2-D Euler code. The use of an adaptive unstructured numerical grid made it possible to obtain a high accuracy of computations and capture regions of induced vorticity for a moderate overall number of mesh points.

The structure of two counter-rotating non-symmetrical vortices was observed after the VDI interaction with a propagating shock wave.

Splitting of the VDI passing through the Prandtl-Meyer expansion wave was studied numerically. The main difference between the interaction of the VDI with a shock wave and an expansion fan is a gradual increase of the induced vorticity.

A method for the computation of an overall induced circulation as a result of the interaction with a number of weak waves was proposed. A simplified approximation for computing this value was derived and its accuracy was estimated analytically. This expression was used for the computation of the expansion angle of the Prandtl-Mayer wave for a given value of the induced circulation.

The obtained shapes of the VDI after the interaction with an expansion wave are close to those obtained for the shock-VDI interactions for the same values of initial and induced circulations. This gives us the opportunity to obtain VDI shapes in a complicated geometry by the simulation of the interaction with a straight propagating shock wave in a rectangular computational domain.

\section{Acknowledgments}

The authors are grateful to Professor P.A. Voinovich and Mr. A.O. Galyukov for a kind permission to use their 2-D Euler unstructured code and unstructured grid generator.

The first author was supported by the British Royal Society and the Israel Academy of Sciences and Humanities to perform the first part of this project at the UMIST-University of Manchester Institute of Science and Technology.

The authors wish to thank Professor M. Leschziner from Department of Mechanical Engineering, UMIST for his hospitality and discussion of this research.

\section{REFERENCES}

[1] F. E. Marble, Gas dynamic enhancement of non-premixed combustion. Hottel plenary lecture, in TwentyFifth Symposium (international) on Combustion, Publish. Combustion Institute, Pittsburgh, 1994, pp 1-12.

[2] I. Waitz, E. Greitzer and C. Tan, Vortices in aero-propulsion systems, in Fluid vortices, Sh. I. Green, ed., Kluwer Academic Publishers, Dordrecht, 1995, pp. 471-532.

[3] J. W. Jacobs, Shock-Induced mixing of light-gas cylinder, Journal of Fluid Mechanics,234 (1992), pp. 629-649. 
[4] J. Yang et al., A model for characterization of a vortex pair formed by shock passage over a light-gas inhomogeneity, Journal of Fluid Mechanics, 258 (1994), pp 217-244.

[5] J. L. Ellzey, M. R. Henneke, J. M. Picone, and E. S. Oran, The interaction of a shock with a vortex: shock distortion and the production of acoustic waves, Physics of Fluids A, 7(1995), pp. 172-184.

[6] C. T. Kao et al., Physical analysis of the two-dimensional compressible vortex-shock interaction, AIAA paper 96-0044, 34th Aerospace Sciences Meeting and Exhibit, January 1996, Reno, NV

[7] J. J. Quirk and S. Karni, On the dynamics of a shock-bubble interaction, Journal of Fluid Mechanics, 318 (1996), pp 129-163.

[8] A. Fursenko, D. Sharov, E. Timofeev, P. Voinovich, Numerical simulation of shock wave interactions with channel bends and gas non-uniformities, Computers and Fluids, 21 (1992), pp 377-396.

[9] A. Fursenko, D. Sharov, E. Timofeev, P. Voinovich, An efficient unstructured Euler solver for transient shocked flows, in Proceedings of the 19 th International Symposium on Shock Waves Held at Marseille, France, June 1993, Shock Waves @Marseille, Springer Verlag, vol. 1 (1995), pp 371-376.

[10] P. Voinovich, Two-dimensional locally adaptive unstructured unsteady Euler code, Advanced Technology Center, St.Petersburg (unpublished), 1993.

[11] A. Galyukov, P. Voinovich, Two-dimensional unstructured grid generator, Advanced Technology Center, St.Petersburg (unpublished), 1993.

[12] R. Fournier, W. Kherrati, VIGIE User manual, version 1.5, INRIA Sophia-Antipolis, France, 1996.

[13] J. Anderson, Fundamentals of Aeradynamics, 2nd edition. McGraw-Hill, New York, 1991. 


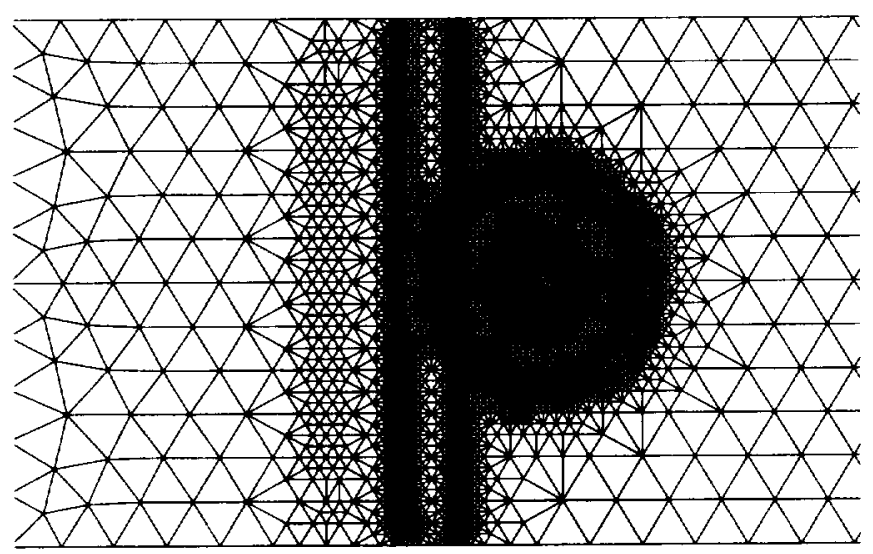

a

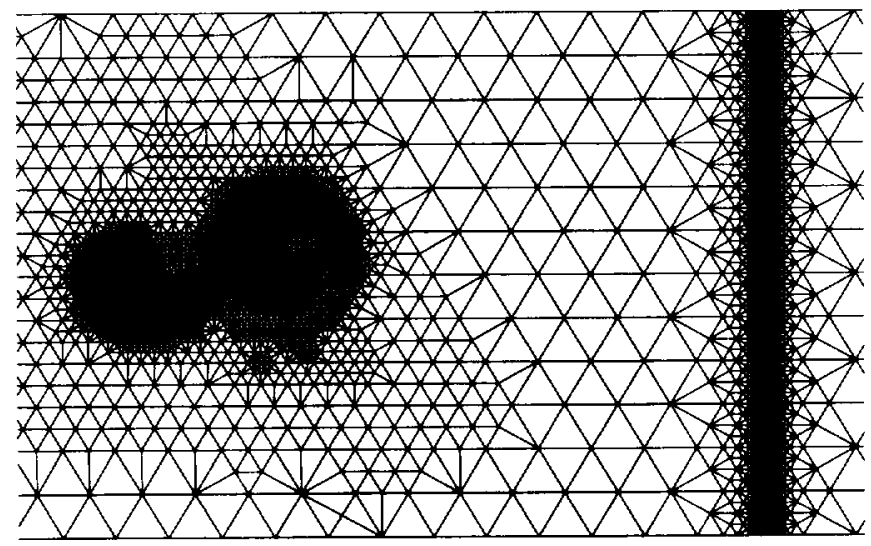

b

FIG. 1. Fragment of unstructured grid uith automatic mesh nefinement: a, before the shock-VDI interaction; $b$, after the interaction 
a

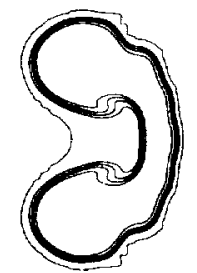

b

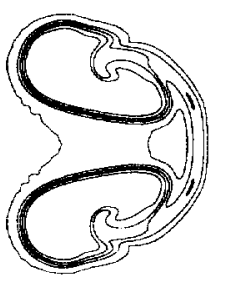

c

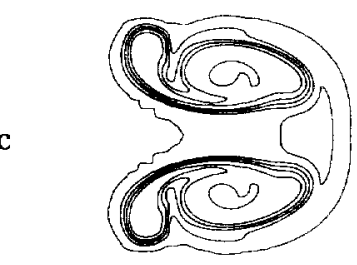

d
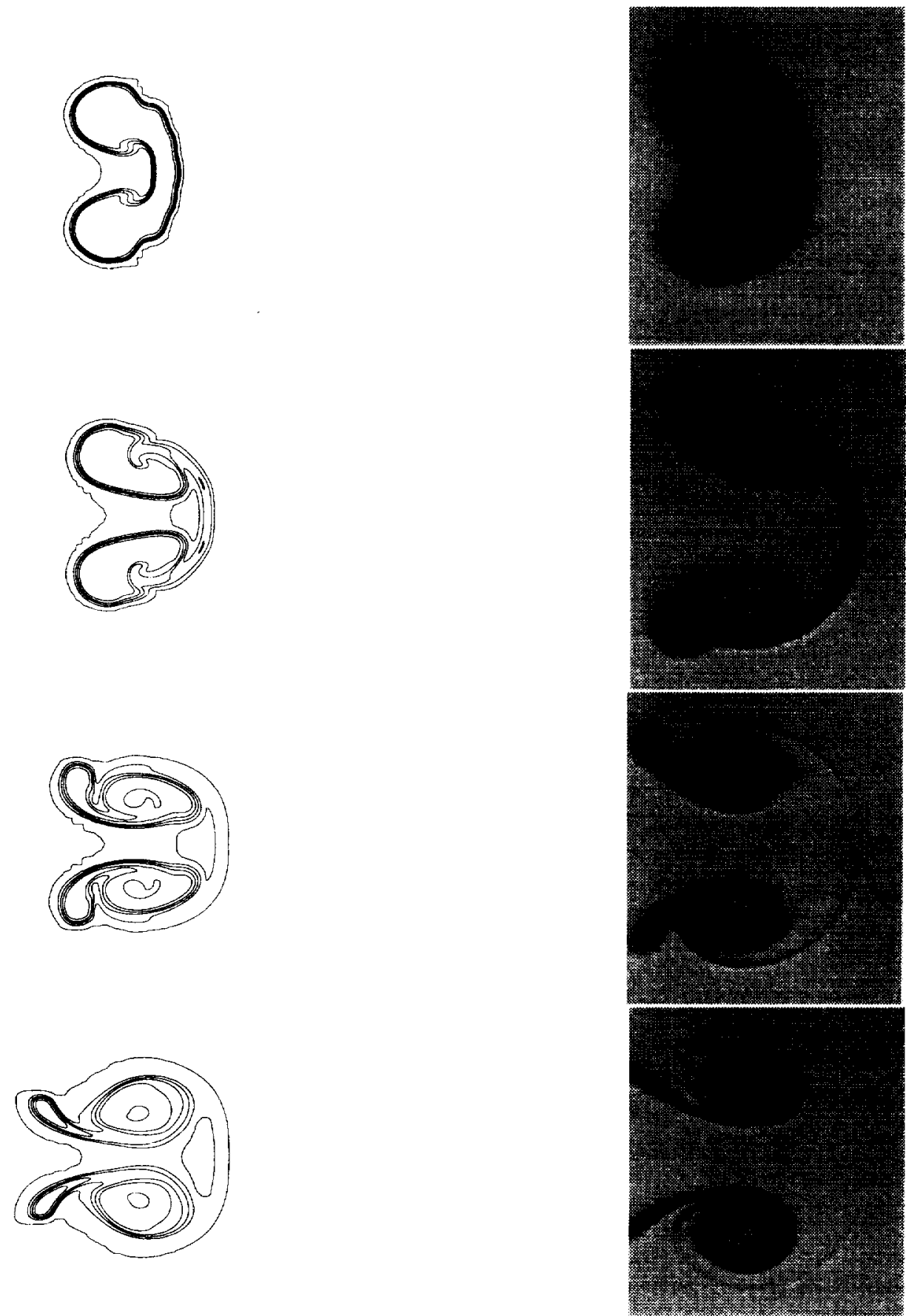

Fic. 2. Interaction of cylindrical bubble urth a propagating shock wave $(M=1.09 ; D=0.14 ; W=0)$. Density isolines are taken with 40 equally spaced intervals between the minimum density inside the bubble and ambient density. The left side-our computational results. The right side-experimental results [3]. Times: a, $123 \mu s ; b, 373 \mu s ; c, 573 \mu s ; d, 773 \mu s$ 

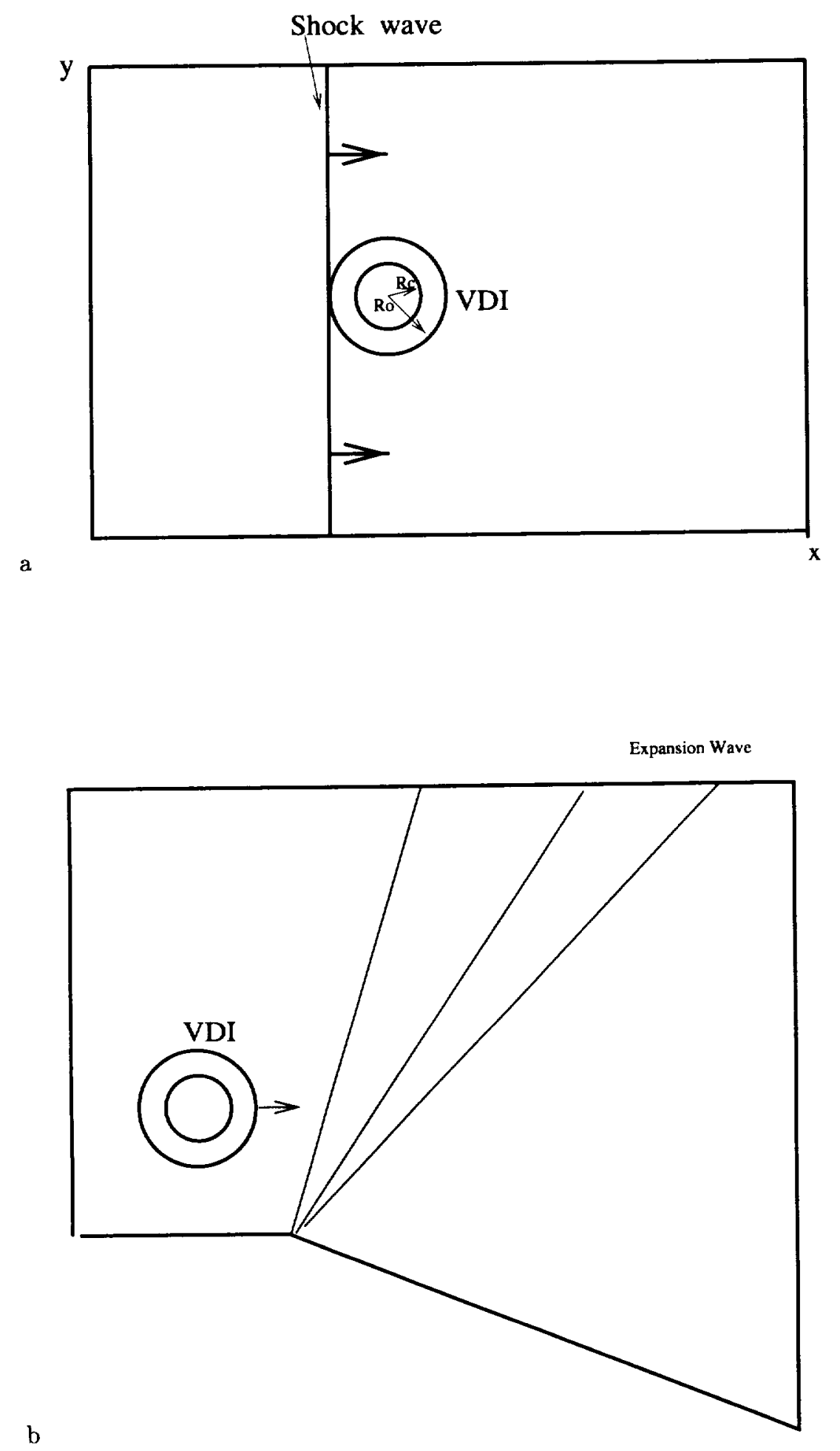

FIG. 3. Computational domain: a, interaction of the VDI with a propagating shock wave; b, interaction of the VDI with the Prandtl-Meyer expansion wave 
a

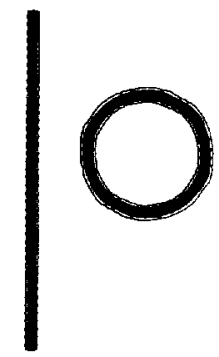

b

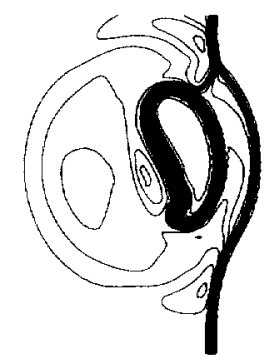

c

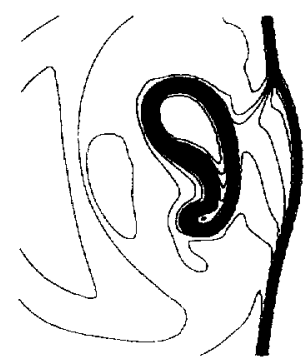

d

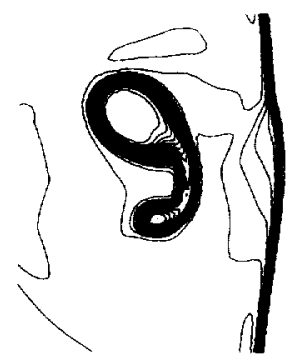

e

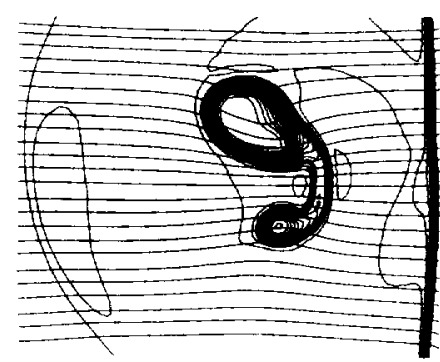

f

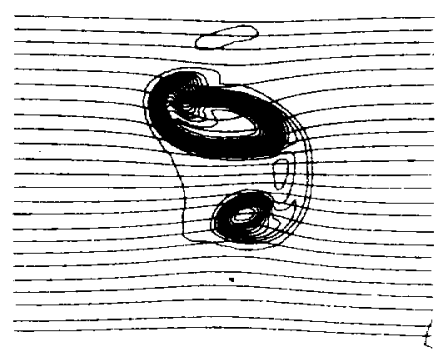

g
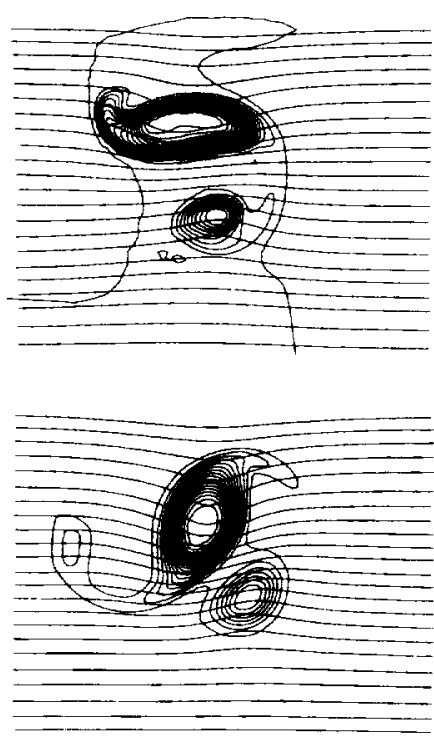

FIG. 4. Interaction of the VDI with a propagating shock wave (D=0.2; $W=0.5)$ Density isolines are taken with 40 equally spaced intervals between density at the center of the counterclockurse vortex and ambient density. Times: $a$, 0 ; $b, 50 \mu s ; c$, $70 \mu s ; d, 90 \mu s ; e, 110 \mu s ; f, 157 \mu s ; g, 203 \mu s ; h, 421 \mu s$ 
a
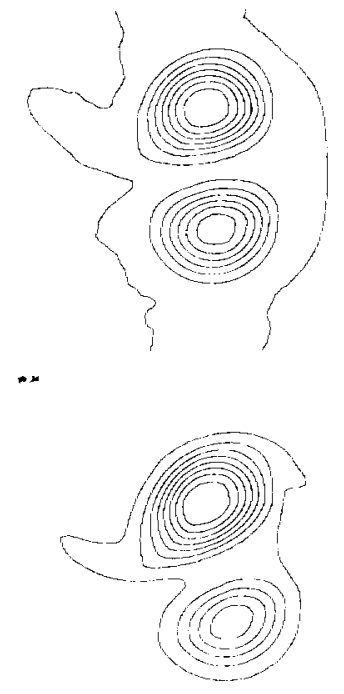

b

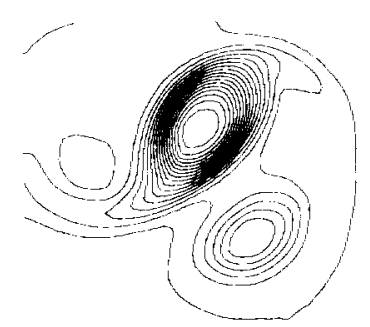

c



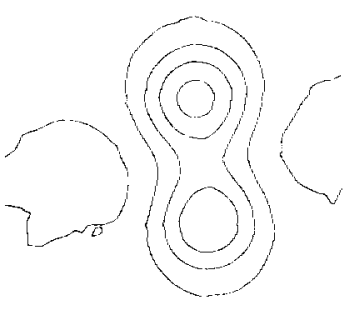

c

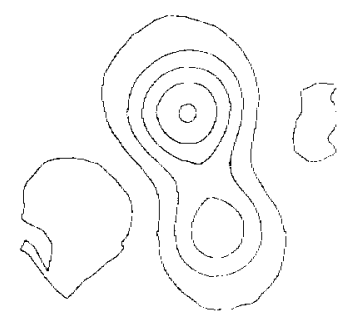

f

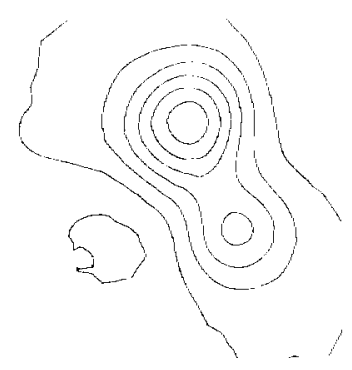

g

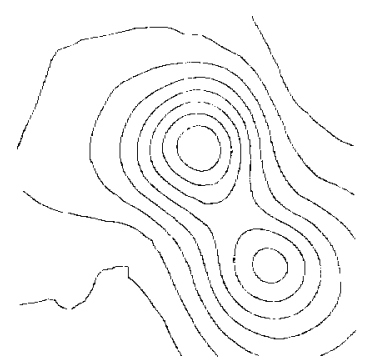

FIG. 5. Patterns of VDI shapes $(D=0.2)$ at $T=421 \mu s: a-d$, density isolines; $e-h$, pressure isolines, a,e, $W=0.1 ; b, f$, $W=0.25 ; c, g, W=0.5 ; d, h, W=1$ 

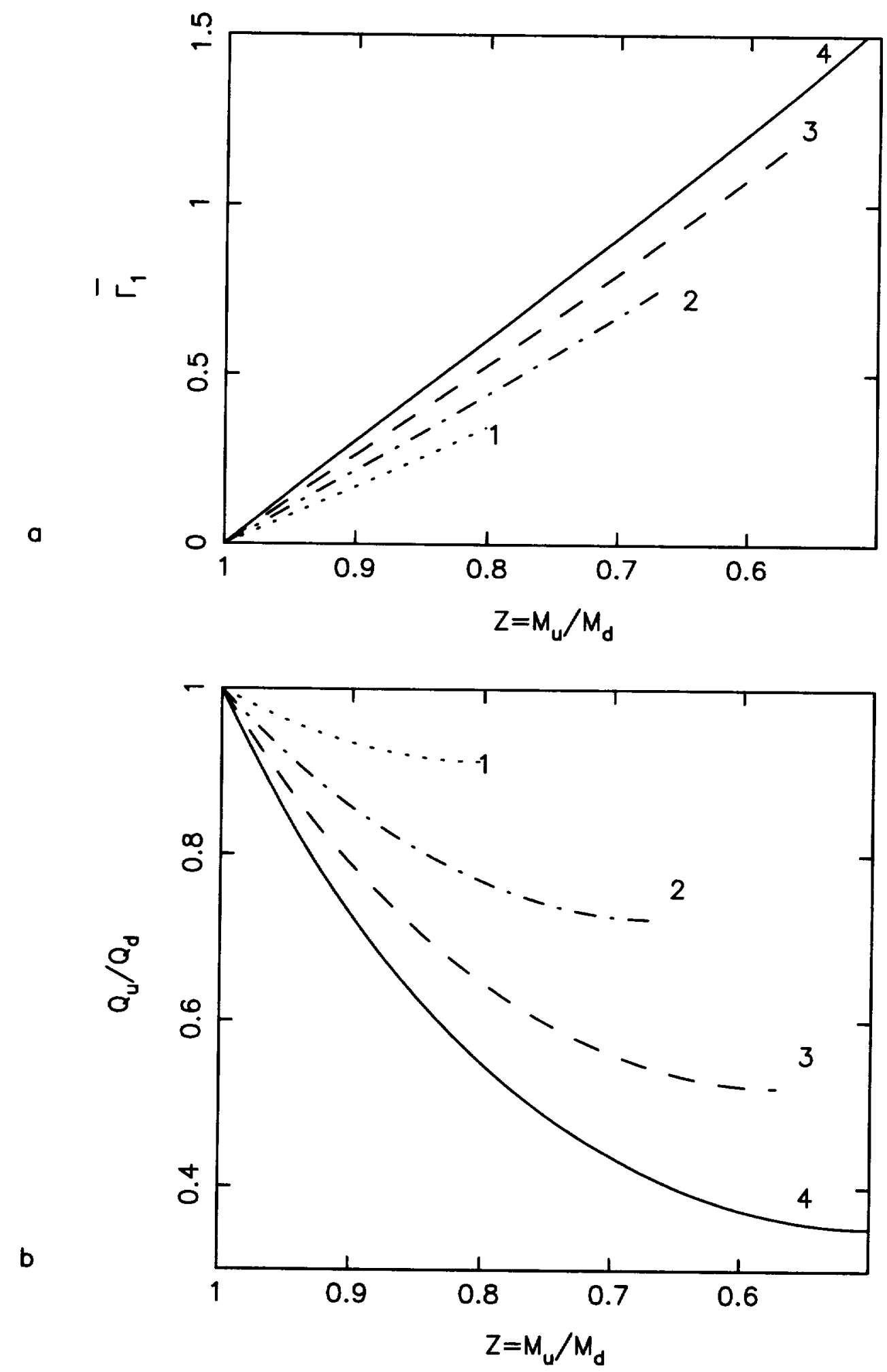

FIG. 6. Estimation of induced cinculation due to the interaction between the VDI and the Prandtl-Meyer expansion wave: a, normalized induced circulation vs $Z=M_{u} / M_{d} ; b$, normalized value of $Q_{u} / Q_{d}$ vs $Z$. Downstream Mach numbers: 1 - 1.25; $2-1.5 ; 3-1.75 ; 4-2.0$ 


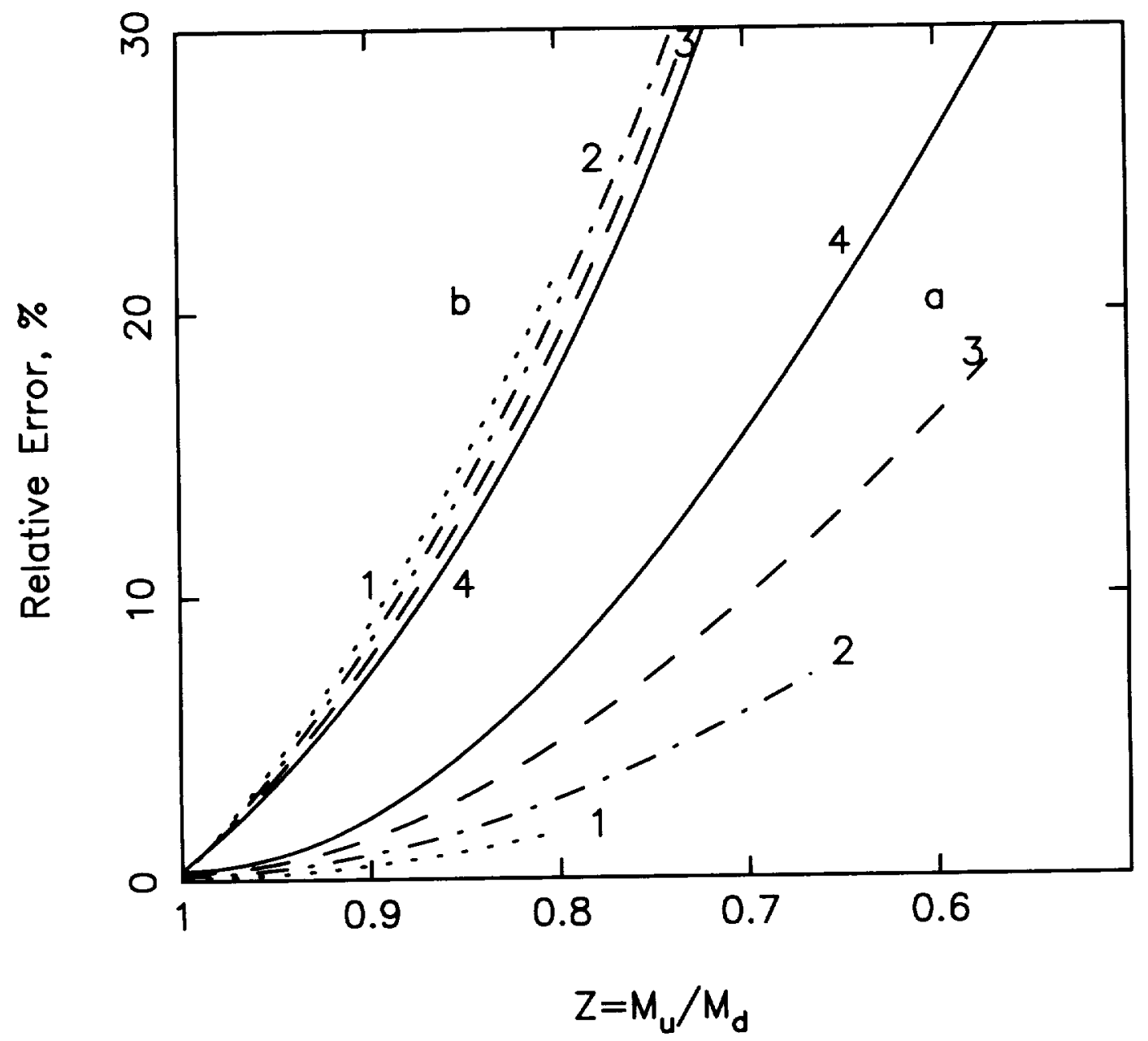

FIG. 7. Relative error of approximation for induced circulation: $a$, by (19); b, by (20). Dounstream Mach numbers: 1 $1.25 ; 2-1.5 ; 3-1.75 ; 4-2.0$ 
a

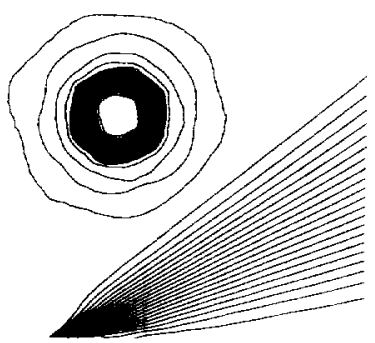

b
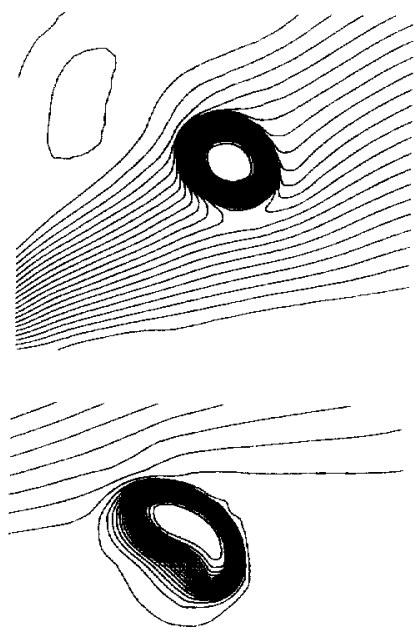

c

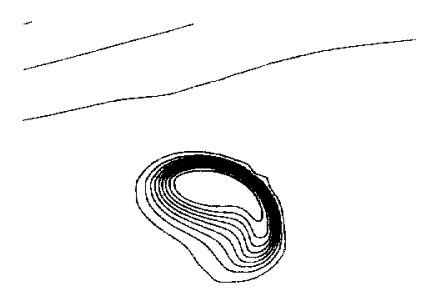

d e

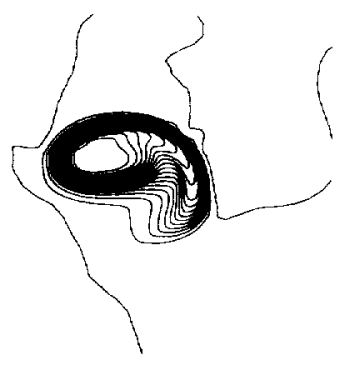

f
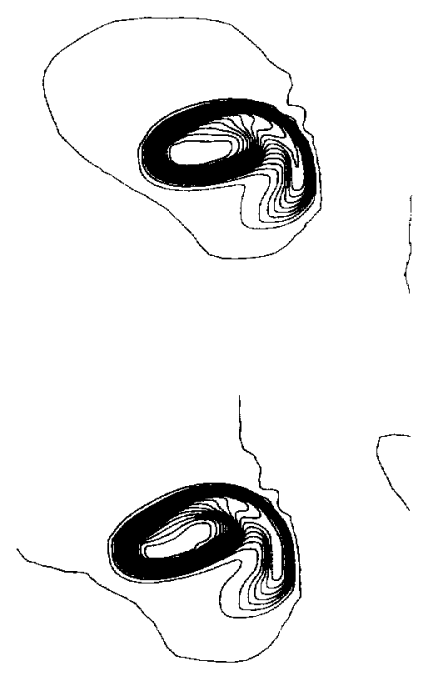

g

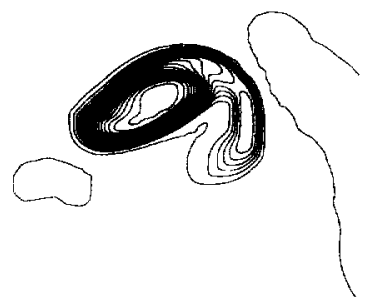

h

FiG. 8. Interaction of the VDI with the Prandtl-Meyer expansion wave (D=0.2). Density isolines are taken with 40 equally spaced intervals between minimum density inside the VDI and density upstream the expansion wave. Times: $a, 0 ; b$ $184 \mu s ; c, 371 \mu s ; d, 453 \mu s ; e, 535 \mu s ; f, 617 \mu s ; g, 699 \mu s ; h, 781 \mu s$ 



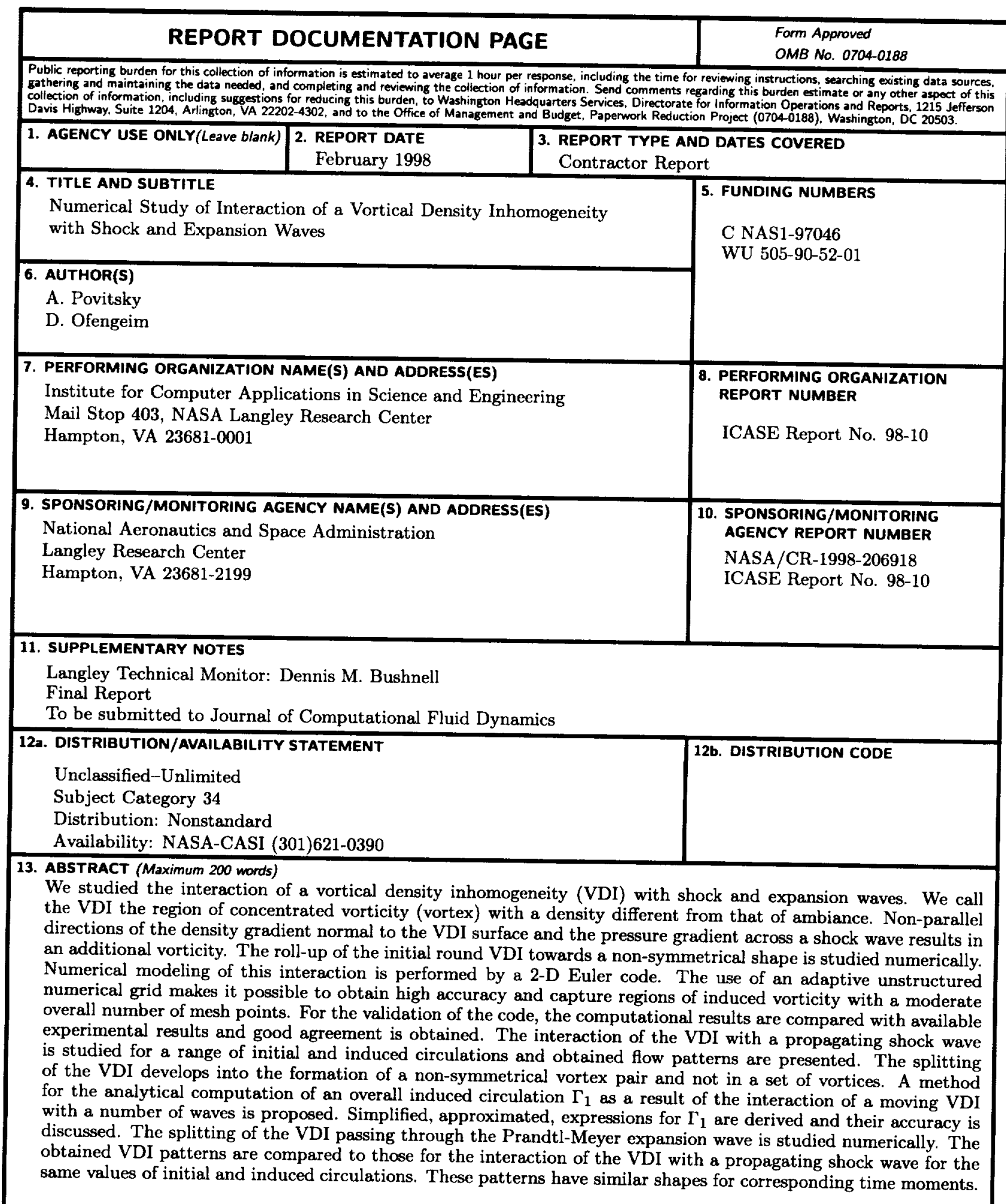

\begin{tabular}{|c|c|c|c|}
\hline \multirow{2}{*}{\multicolumn{3}{|c|}{$\begin{array}{l}\text { 14. SUBJECT TERMS } \\
\text { vortices, unstructured grids, automatic mesh refinement, Euler equations, vortical } \\
\text { density, inhomogeneity, shock wave, expansion wave }\end{array}$}} & \begin{tabular}{|c|} 
15. NUMBER OF PAGES \\
23
\end{tabular} \\
\hline & & & $\begin{array}{r}\text { 16. PRICE CODE } \\
\text { A03 }\end{array}$ \\
\hline $\begin{array}{l}\text { 17. SECURITY CLASSIFICATION } \\
\text { OF REPORT } \\
\text { Unclassified }\end{array}$ & $\begin{array}{l}\text { 18. SECURITY CLASSIFICATION } \\
\text { OF THIS PAGE } \\
\text { Unclassified }\end{array}$ & $\begin{array}{l}\text { 19. SECURITY CLASSIFICATION } \\
\text { OF ABSTRACT }\end{array}$ & $\begin{array}{l}\text { 20. LIMITATION } \\
\text { OF ABSTRACT }\end{array}$ \\
\hline ISN 7540-01-280-5500 & & & $\begin{array}{l}\text { Standard Form 2987(Rev. } 2 \\
\text { Prescribed by ANSI Std. Z39-18 } \\
\text { 298-102 }\end{array}$ \\
\hline
\end{tabular}

